\title{
118. LAS COMUNIDADES CON CACTÁCEAS DEL SUR DEL PERÚ, II. NUEVA ASOCIACIÓN Y ALIANZA DEL DESIERTO PACÍFICO
}

\author{
Antonio GALÁN DE MERA, César CÁCERES y Adolfo GONZÁLEZ
}

The cactus communities in Southern Peru, II. A new association and alliance of the Pacific Desert.

Palabras clave. Fitosociología, Cactaceae, Perú, Neoporteria, Haageocereus.

Key words. Phytosociology, Cactaceae, Peru, Neoporteria, Haageocereus.

Dentro del programa que estamos llevando a cabo sobre la sintaxonomía de la vegetación del Perú (Galán de Mera, 1999), nos encontramos describiendo sobre todo las asociaciones y unidades superiores del sur, en los departamentos de Arequipa, Moquegua y Tacna (fig. 1), como continuación hacia el norte de los resultados fitosociológicos obtenidos por otros autores en Chile (Ruthsatz, 1995) y Bolivia (Navarro, 1993).

En el Perú, la costa bañada por el océano Pacífico, entre los departamentos de Ica y Tacna, muestra los relieves de la cordillera externa que preceden a los Andes, algunos de ellos muy próximos al mar y con una buena cantidad de endemismos que determinan el sector Arequipeño de la provincia fitogeográfica Limeño-Ariqueña (Müller, 1985; Galán de Mera et al., 1997). Estos promontorios externos están muy próximos al mar y pueden llegar a alcanzar hasta $950 \mathrm{~m}$ de altitud reteniendo la nubosidad que se forma como consecuencia del paso de la corriente fría de Humboldt, y favoreciendo precipitaciones que permiten el desarrollo de comunidades de Cactáceas similares a las que se presentan en las laderas occidentales andinas (Arévalo del Carpio, 1993; Lombardi, 1994; Galán de Mera y Vicente Orellana, 1996). La separación fitogeográfica entre las laderas andinas termo-mesotropicales áridas y la costa termomediterránea hiperárida del Pacífico
(Galán de Mera y Gómez Carrión, 2001) posibilita la existencia de nuevas asociaciones. La nomenclatura y autoría de los táxones coincide con Brako y Zarucchi (1993).

Neoporterio islayensis-Neoraimondietum arequipensis Galán de Mera, Cáceres y González ass. nova

[Tabla 1, sintypus: inv. 1]

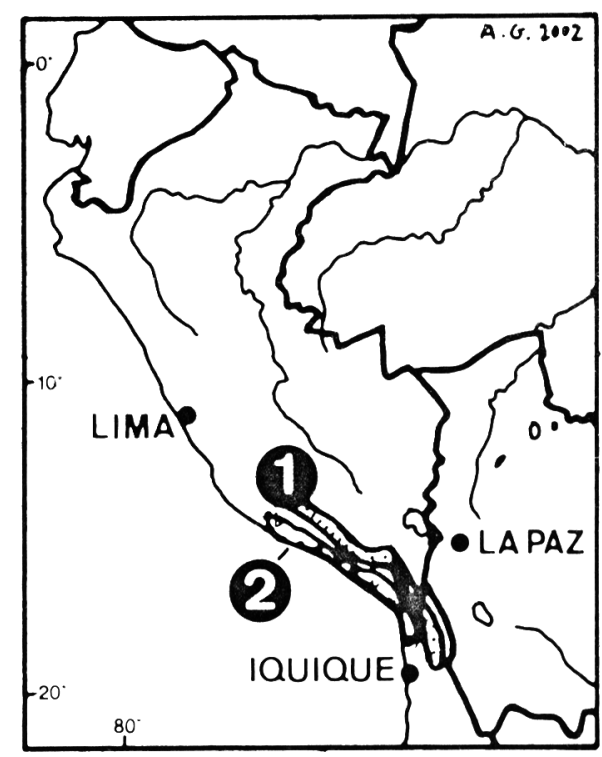

Figura 1. Distribución de las dos alianzas de comunidades de Cactáceas descritas en el sur del Perú. 1: Corryocaction brevistyli; 2: Haageocerion australis. Distribution of the two alliances of cactus communities described in Southern Peru. 
Comunidades de Cactáceas dominadas por el «gigante» (Neoraimondia arequipensis), que se desarrollan sobre promontorios rocosos con fuertes pendientes (litosoles y arenosoles), y constituidos por materiales plutónicos cretácicos, metamórficos precambrianos y facies marinas volcánico-sedimentarias jurásicas (IGM, 1975), bajo termoclima termomediterráneo hiperárido.

En su composición florística cabe destacar a Corryocactus brachypetalus, Neoporteria islayensis y Haageocereus australis, lo que permite diferenciar esta asociación de las de la alianza Corryocaction brevistyli, que además guardan una mayor diversidad en su composición florística (Galán de Mera y Gómez Carrión, 2001). Tanto Corryocactus brachypetalus como Neoporteria islayensis son endemismos de la costa sur peruana (Britton y Rose, 1937; Hunt, 1992), con una disyunción del segundo en el centro (Ostolaza y Mischles, 1984). Sin embargo, Haageocereus australis está citado tanto en la costa peruana (Galán de Mera, 1995) como chilena (Ritter, 1980, 1981) lo que sugiere el areal de la nueva alianza Haageocerion australis all. nova (fig. 1). Aunque Neoporterio-Neoraimondientum (tipo de la alianza) es una fitocenosis pobre en especies, la presencia de Neoraimondia arequipensis y Grindelia glutinosa nos lleva a la inclusión de la alianza Haageocerion australis dentro del orden Oreocereo leucotrichi-Neoraimondietalia arequipensis de la clase Opuntietea sphaericae.

Asimismo, esta nueva asociación es la etapa climácica de la serie termomediterránea limeño-ariqueña hiperárida acidófila del gigante- Neoporterio islayensisNeoraimondieto arequipensis sigmetum, de la cual sólo hemos podido observar una etapa de sustitución constituida por pastizales terofíticos con Cristaria multifida y Tiquilia litoralis, típicos de las lomas costaneras del desierto peruano-chileno (Ferreyra, 1953).

Tabla 1

Neoporterio islayensis-Neoraimondietum arequipensis Galán de Mera, Cáceres y González ass. nova (Haageocerion australis, Oreocereo-Neoraimondietalia arequipensis, Opuntietea sphaericae)

\begin{tabular}{lcccccc}
\hline Inventario $\mathrm{n}^{\circ}$ & 1 & 2 & 3 & 4 & 5 & 6 \\
Área $^{2}$ & 100 & 100 & 50 & 100 & 100 & 100 \\
Altitud $(\mathrm{m})$ & 250 & 200 & 250 & 250 & 250 & 280 \\
Orientación & $\mathrm{SE}$ & $\mathrm{O}$ & $\mathrm{NE}$ & $\mathrm{O}$ & $\mathrm{SE}$ & $\mathrm{O}$ \\
Inclinación $\%$ & 80 & 60 & 80 & 60 & 80 & 50
\end{tabular}

Características de asociación y unidades superiores

Neoraimondia arequipensis

Corryocactus brachypetalus

Grindelia glutinosa

Neoporteria islayensis

Haageocereus australis

$\begin{array}{lll}3 & \\ 1 & 2 \\ + & + \\ + & +\end{array}$

2
2
+
+
+

$\begin{array}{llll}2 & 2 & 3 & 2 \\ 1 & 1 & + & 1 \\ + & 1 & + & 2 \\ 1 & + & . & + \\ + & + & + & +\end{array}$

\section{Compañeras}

Trixis cacalioides

Tiquilia litoralis

Nolana lycioides

Localidades.- I a 5: Morro de Sama (Tacna), 6: Punta de Islay (Arequipa). 


\section{ESQUEMA SINTAXONÓMICO}

\section{OPUNTIETEA SPHAERICAE Galán de Mera y Vicente Orellana 1996 \\ + Oreocereo leucotrichi-Neoraimondietalia arequipensis Galán de Mera y Vicente Orellana 1996 \\ * Haageocerion australis Galán de Mera, Cáceres y González all. nova}

1. Neoporterio islayensis-Neoraimondietum arequipensis Galán de Mera, Cáceres y González ass. nova [Comunidades de Cactáceas columnares termomediterráneas hiperáridas del sur del Perú y norte de Chile].

AGRADECIMIENTOS. Deseamos expresar nuestro agradecimiento a la Escuela de Postgrado de la Universidad Nacional Mayor de San Marcos por las facilidades otorgadas tanto en los trabajos de campo como en el herbario USM. Este trabajo ha sido realizado gracias a una beca del Programa de Cooperación Interuniversitaria de la Agencia Española de Cooperación Internacional (AECI).

\section{BIBLIOGRAFÍA}

ARÉVALO DEL CARPIO, C. -1993- Visita a los alrededores de la ciudad de Arequipa y punta de Corio (Islay). Quepo 7: 46-55.

BRAKO, L. y J.L. ZARUCCHI - 1993-Catálogo de las Angiospermas y Gimnospermas del Perú. Missouri Botanical Garden. St. Louis.

BRITTON, N.L. \& J.N. ROSE - 1937-The Cactaceae. Descriptions and illustrations of plants of the cactus family. Dover Publications. New York.

FERREYRA, R. - 1953- Comunidades vegetales de algunas lomas costaneras del Perú. Bol. Estac. Exp. Agric. "La Molina" 53: 1-88.

GALÁN DE MERA, A. -1995- Nuevas áreas y localidades para plantas del Perú. Arnaldoa 3(1): 45-49.

GALÁN DE MERA, A. -1999- Las clases fitosociológicas de la vegetación del Perú. Bol. Lima 117: 84-98.

GALÁN DE MERA, A. y J. GÓMEZ CARRIÓN -
2001- Las comunidades con Cactáceas del sur del Perú. Nuevos datos sobre la alianza Corryocaction brevistyli. Acta Bot. Malacitana 26: 240-246.

GALÁN DE MERA, A. y J.A. VICENTE ORELLANA -1996- Las comunidades con Corryocactus brevistylus del sur del Perú. Phytologia 80(1): 40-47.

GALÁN DE MERA, A., J.A. VICENTE ORELLANA, J.A. LUCAS GARCIA \& A. PROBANZA LOBO - 1997- Phytogeographical sectoring of the Peruvian coast. Global Ecol. Biogeogr. Lett. 6: 349-367.

HUNT, D. -1992-CITES Cactaceae Checklist. Royal Botanic Gardens Kew \& International Organization for Succulent Plant Study. Whitstable.

IGM (Instituto de Geología y Minería) - 1975-Mapa geológico del Perú (1: 1.000.000). Lima.

LOMBARDI, A. - 1994- Expedición al sur del Perú. Quepo 8: 49-62.

MÜLLER, G.K. -1985- Zur floristichen Analyse der peruanischen Loma-Vegetation. Flora 176: 153-165.

NAVARRO, G. -1993- Vegetación de Bolivia: el Altiplano meridional. Rivasgodaya 7: 69-98.

OSTOLAZA, C.N. y T.C. MISCHLES - 1984- Islaya omasensis. Bol. Lima 6(32): 63-65.

RITTER, F. -1980- Kakteen in Südamerika. Band 3. Chile. Spangenberg.

RITTER, F. -1981- Kakteen in Südamerika. Band 4. Peru. Spangenberg.

RUTHSATZ, B. -1995- Vegetation und Ökologie tropischer Hochgebirgsmoore in den Anden Nord-Chiles. Phytocoenologia 25(2): 185-234.

Aceptado para su publicación en febrero de 2002

Dirección de los autores. A. GALÁN DE MERA y A. GONZÁlEZ: Departamento de Ciencias Ambientales y Recursos Naturales, Laboratorio de Botánica, Universidad San Pablo-CEU, Apartado 67, E-28660- Boadilla del Monte, Madrid, España, E-mail: agalmer@ceu.es; C. CÁCERES: Departamento de Botánica, Facultad de Biología, Universidad Nacional «Jorge Basadre Grohmann», Tacna, Perú. 